\title{
$\mathrm{u}$-Healthcare 환경에서의 효율적인 ECG 데이터 전송 방안에 관한 연구
}

\author{
조균연 ${ }^{*}$, 이서준 ${ }^{* *}$, 이태로 ${ }^{* * *}$
}

고려대학교 BK21+프로그램, 연구교수, 고려대학교 BK21+ 프로그램, 일반대학원 보건과학과*, 고려대학교 BK21+ 프로그램, 보건행정학과 교수

\section{Research on a Solution for Efficient ECG Data Transmission in u-Healthcare Environment}

\author{
Gyoun-Yon Cho*, Seo-Joon Lee ${ }^{* *}$, Tae-Ro Lee ${ }^{* * * *}$ \\ Korea University, Research Professor, BK21+ Program, Department of Public Health Sciences \\ Graduate School* \\ Korea University, Department of Healthcare Management, BK21+ Program, Department of Public \\ Health Sciences Graduate School ${ }^{* *}$ \\ Korea University, Professor, Department of Healthcare Management, BK21+ Program, Department of \\ Public Health Sciences Graduate School ${ }^{* * * *}$
}

요 약 u-Healthcare 환경에서는 많은 양의 중요한 의료정보가 무선을 통해 처리된다. 따라서 데이터를 전송하는 네 트워크 시스템의 효율 개선이 필요하다. 이 논문은 u-Healthcare 환경에서 ECG 데이터를 효율적으로 전송하기 위한 압축 솔루션(ECGLZW)을 제시한다. 실험 결과, ECGLZW의 평균 압축률은 4.6으로써, 기존의 압축 방식(Huffman 압 축 방식과 LZW 압축 방식)에 비해 $200 \%$ 정도 효율이 개선됨을 알 수 있다. ECGLZW의 높은 압축률로 무선채널의 효율이 개선되고, 이를 네트워크 오류에 대한 재전송 및 암호화에 활용함으로써 데이터에 대한 신뢰성 및 $\mathrm{u}$-Healthcare 정보에 대한 보안을 강화할 수 있다.

주제어 : u-Healthcare 환경, ECG, 압축 솔루션, ECGLZW, 무선통신

\begin{abstract}
In u-Healthcare environment, large amounts of important medical information is processed through wireless communication. Therefore there is a need to increase the efficiency of the network system of sending ECG data. This paper presents a compression solution for efficient ECG data transmission(ECGLZW) in u-Healthcare environment. The results showed that the average compression ratio of ECGLZW was 4.6, which got $200 \%$ better than existing methods(Huffman and LZW compression). ECGLZW's high compression ratio can increase the efficiency of wireless channels. As a result, reliable communication and security of u-Healthcare information could be achieved by applying these remaining channels to retransmission and encryption.
\end{abstract}

Key Words : u-Healthcare environment, ECG, compression solution, ECGLZW, wireless communication

Received 13 December 2013, Revised 13 January 2014 Accepted 20 January 2014

Corresponding Author: Tae-Ro Lee(Korea University, Dept of Healthcare Management Professor)

Email: trlee@korea.ac.kr

ISSN: $1738-1916$
(C) The Society of Digital Policy \& Management. All rights reserved. This is an open-access article distributed under the terms of the Creative Commons Attribution Non-Commercial License (http://creativecommons.otg/licenses/by-nc/3.0), which permits unrestricted non-commercial use, distribution, and reproduction in any medium, provided the original work is properly cited. 


\section{Introduction}

Nowadays it is hard to find any healthcare facilities that do not depend on wired or wireless communication. This development in communication has leveled the playing field of medical areas and has now enabled patients to monitor their health status anytime and anywhere[1], [2]. In fact, in some communication advanced areas, even remote medical examination and treatment is already possible. Such environment is so called $\mathrm{u}$-Healthcare(Ubiquitous Healthcare) environment, where providing healthcare service, registering for a reservation, detecting emergency, are all automatically processed through wireless communication.

However, there are some specific but critical problems of medical information systems striving for $\mathrm{u}$-Healthcare today. One of them is that the network system of sending ECG(Electrocardiography) data of patients being inefficient. Indeed this is a critical problem when considering the importance of ECG data which is the core and representative information of any patient's status. Prior research have already pointed out that in a typical emergent situation, ambulance paramedics still use a cellular network to communicate with the emergency center because of the lack of technology in sending the ECG data through the network[3].

For this reason, we present a solution for efficient ECG data transmission in $\mathrm{u}$-Healthcare environment. Among a variety of methods to achieve efficient transmission our research team chose to focus on the compression rate of the ECG data, so that the data could be sent as fast as possible. Through this, even if errors do occur, the communication's restoration time will be cutdown. This is possible because the data packet size has been compressed so that it could be transmitted more frequently, thereby increasing the chance of successful transmission.

In this paper is presented not only the optimized compression algorithm for ECG data, but also the overall system architecture of how our solution should be applied. Our results have proved to be better in compression rate in regard to ECG data than other recent compression methods available today.

\section{Related Research}

\subsection{Related Researches in Data Processing}

Any data may be intended for some purpose but for most of the time the data includes other information beyond that intended purpose. Thus, data processing can be of use when extracting only the valuable information in that data, and especially, in a very large dataset. In the same sense for example, compressing or coding a data is also a sub form of data processing.

There are many sorts of recent researches that put their focus on compression for a diversity of purposes. Some research used wavelet for compressing medical data[4], [5] under the implication that the effectiveness of the medical database in healthcare facilities is expected to be enhanced. Another research concentrated on lossless compression of medical images[6] which was quite radical considering the fact that most methods today choose to minimize images through lossy compression. Their research significance was that it could increase the efficiency of PACS(Picture Archiving Communication System) in medical fields.

\subsection{ECG Data}

ECG(Electrocardiography) measures the electrical activity of the heart. When the heart pumps, it produces a few electrical pulses which spread out through the body. These tiny electric signals are analog signals which can be detected by ECG machines. ECG signals are converted to digital signals and are then usually shown in a simple graph, as shown in Figure 1[6]. 


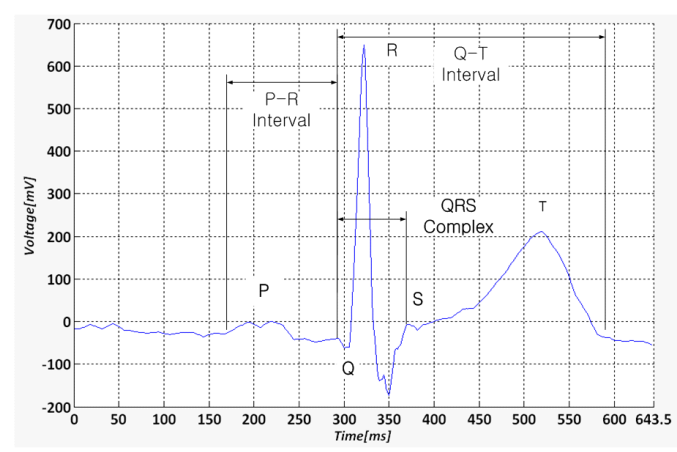

[Figure 1] Typical ECG Signal

ECG is a strong indicator of the rate and regularity of the heartbeat. Medical professionals infer any damage to the heart through ECG such as the effects of drugs or devices that disturb normal heartbeat. Therefore it is important to cautiously analyze the pattern and form of the signal when giving consideration to transmitting ECG data in order to avoid distortion. Related research presents an adaptive morphological filter developed using multiscale mathematical morphology to reject broadband noise from ECG signals without affecting the feature waveforms[7]. Another study presented various novel block based time-frequency domain adaptive filter structures for ECG enhancement for remote health monitoring systems[8].

\subsection{Digital Data Compression}

Any kind of data can be compressed. Basically, the main idea is to eliminate the redundancy in that data, so the overall data size can be reduced. The most representative methods in digital data compression are Huffman coding and LZW(Lempel-Ziv Welch) coding, which will be explained below.

Huffman coding algorithm is a probability coding method which decides the code size by the probabilities of individual data symbols[9], [10].

LZW is a dictionary coding variant of Lempel-Ziv Compression[11], [12] and is the most commonly used algorithm in practice. The method is widely used in
Unix file compression and in GIF image compression. The most representative example is the compression software Zip, which is favored by any computer users nation-wide.

These are all lossless compression algorithms, which are preferable in healthcare fields where information of the patient is valuable.

\section{System Overview}

\subsection{Overall Architecture}

This section shows the overall architecture of the ECG data transmission system to explain where our proposed solution will be placed. The compression algorithm ECGLZW (Electrocardiography LZW) that we have developed is based on the principles of LZW, and is specialized for ECG data. In short, our solution will act as a medium in the $\mathrm{u}$-Healthcare network

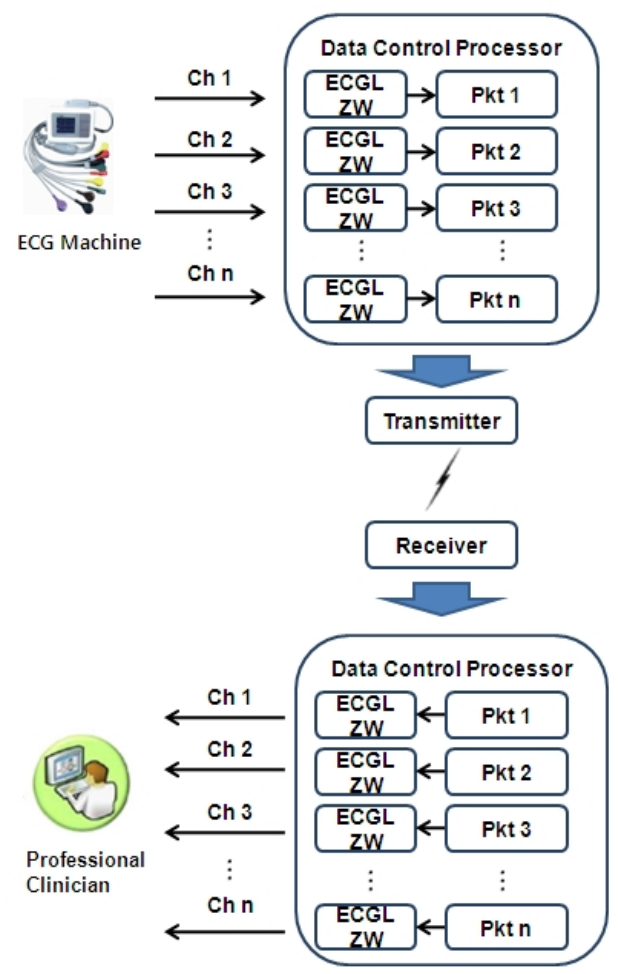

[Figure 2] Overall Architecture 
between the patient and the personal computer of the medical professionals, as shown in Figure 2.

In order to monitor the vital sign of the patient, the patient is first put in bed with the ECG machine aside. Small ECG leads are then attached to the patient. Although it is recommended as a standard medical protocol to ensure 12 ECG leads to be attached to the patient for detailed analysis of the patient's vital sign, the number of ECG leads can vary as long as accurate ECG measurement can be ensured. Each ECG lead channels sense vital signs of the patient, and raw ECG data is gathered.

Normally, this raw ECG data will simply be shown in a display by the ECG machine. However, our general idea is to first compress the data by our developed DCP(Data Control Processor), and send it through the network and towards the final destination which is most likely to be the professional clinician's main PC. The ECG data is compressed to minimize data errors and maximize transmission speed. Before the coded data arrives to the clinician, it is decoded by the DCP before being shown in the main computer's GUI(Graphic User Interface).

\subsection{The Compression Algorithm}

The developed compression algorithm is coded in the DCP. Programming was conducted using C Language.

Normally, ECG machine has 1 to 12 multiple ECG leads that samples the data packet by simultaneously collecting ECG samples continuously from each ECG leads. In other words, the first byte of the packet is consisted of all the samples collected in the multiple ECG leads continuously.

For instance, 'Packet 1' would consist of time stamp t1, $-108,-804,266$, time stamp t2, and -196 which is the capacity limit of the packet. The next packet 'Packet 2' would consist of -804, 210, time stamp t3, $-349,-804$, and 210. Refer to Figure 3 for better description.

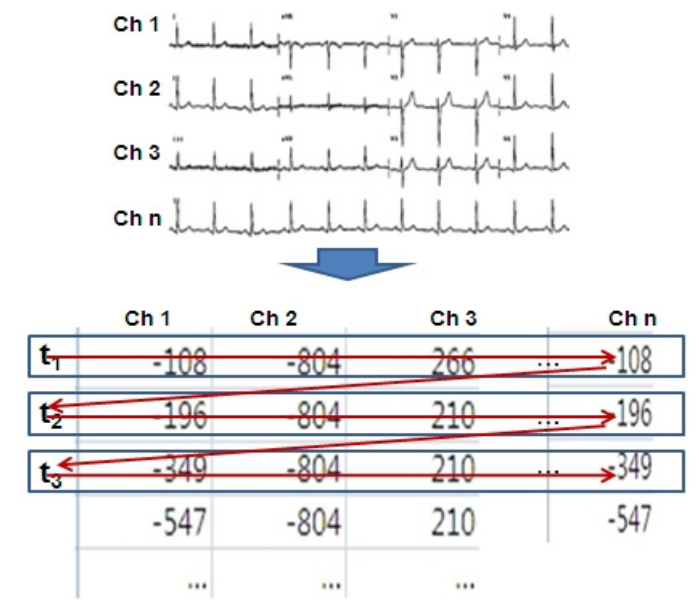

[Figure 3] Typical ECG Data Transmission

However, ECGLZW is different. Once the data is handed over to the DCP, the DCP divides the samples by $n$ channels. Then, for each $n$ channel, packet $m$ is consisted of the compression data of samples within 2 seconds. The reason for making the packet data by 2 seconds is because such time interval is optimal for efficient and real-time data transmission(Figure 4).

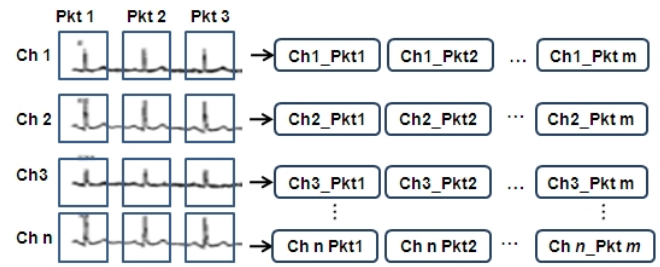

[Figure 4] ECGLZW Data Transmission

In short, the ECGLZW is a newly proposed algorithm in which a channel dividing preprocessing algorithm is applied before LZW compression. This innovative and rather fundamentally different algorithm, the ECGLZW algorithm, proved to significantly enhance the compression ratio than any widely used compression algorithms. The specific evaluation results will be shown in section 4 . 


\section{Evaluation}

LZW compression and Huffman compression methods were selected as ECGLZW's comparisons as they are still the most widely used compression methods today. We did not conduct a comparison analysis between prior researches related to ECG data compression because almost all of the prior researches were based on lossy algorithms. We used the data samples provided from Physionet[13] to compare the mean of compression ratio between the three methods. Compression ratio is related by

$$
\text { Compression Ratio }=\frac{\text { Uncompressed Size }}{\text { Compressed Size }}
$$

and is expressed in real numbers(e. g. 1.66 or 2.009). We used two data sample groups; MIMIC(Multiparameter Intelligent Monitoring in Intensive Care) data and UCD(University College Dublin) data. The MIMIC data represents data recorded from ICU(Intensive Care Unit) patients. We used 30 samples with each samples containing ECG data of 5 minute period. The UCD data contains 25 full overnight polysomnograms with simultaneous three channel Holter ECG, from adult subjects with suspected sleep-disordered breathing. We used 27 samples with each samples containing ECG data of 5 minute period. The statistical software SPSS 21.0 was used for ANOVA(Analysis of Variance).

\section{〈Table 1〉 Comparison of Compression Ratio Among Compression Methods}

\begin{tabular}{ccccc}
\hline & Huffman & LZW & ECGLZW & \\
Var. & $\begin{array}{c}\text { MIMIC n=30, (MIMIC n=30, } \\
\text { UCD n=27) }\end{array}$ & UCD n=27) & UCD n=27) & \\
\hline MIMIC & $1.67 \pm 0.01_{\mathrm{a}}$ & $2.25 \pm 0.02 \mathrm{~b}$ & $4.29 \pm 0.06_{\mathrm{c}}$ & $\mathrm{p}<0.0001$ \\
UCD & $2.01 \pm 0.01$ & $2.63 \pm 0.05$ & $4.91 \pm 0.22$ & $\mathrm{p}<0.0001$ \\
\hline
\end{tabular}

Means in a row with different superscript letters are significantly different, $\mathrm{p}<0.05$ by ANOVA and Duncan's test(Kruskal-Wallis test for UCD).
For UCD data, Kruskal-Wallis test was used because samples were lower than 30. In its final analysis, ECGLZW proved to significantly enhance the compression ratio of the data packet than Huffman compression or LZW compression. In case of MIMIC data, the compression ratio of Huffman compression and LZW compression was only 1.66 and 2.2478 respectively, whereas our compression method's compression ratio was 4.2929. This number is almost double the compression ratio than any of the compared two methods.

Results were similar to UCD data also, in which Huffman compression and LZW compression was only 2.009 and 2.6293 respectively. On the other hand, ECGLZW compression method showed a compression ratio of 4.9071. Overall, ECGLZW compression method's compression ratio was 4.6.

\section{Conclusion}

People's perception is shifting towards patientoriented healthcare from the classical, hospital centered healthcare, which has been propelling the evolution of telemedicine research from the classic e-Health[14] to $\mathrm{u}$-Healthcare[15]. However, recent limitation of $\mathrm{u}$-Healthcare was that most of the data in wireless communication were not processed before being transmitted in $\mathrm{u}$-Healthcare environment. Of course, headers specific to the network environment(e. g. Wi-Fi or Bluetooth) are attached to the data packet, but this cannot be seen as data processing but simply a procedure for communication protocol.

Therefore, this paper presented a research on a solution for efficient ECG data transmission in $\mathrm{u}$-Healthcare environment.

With ECGLZW's high compression ratio, empty channels will become available. Since our results showed that ECGLZW's compression ratio was 4.6 in average, approximately $80 \%$ of the channels will 
become available. These remaining channels could be used for retransmission and encryption. Consequently, it leads to higher reliability of transmission.

Further research is needed for ECGLZW to be embedded to mobile or ad hoc network[16], [17] because our research program is only compatible for Microsoft Windows OS(Operation System).

\section{References}

[1] Lee SJ, Lee TR. Design of Remote Infusion Pump Monitoring System Using Wireless Network and RFID Technology. The Journal of Digital Policy \& Management. 2013.

[2] Lee SJ, Sim HJ, Lee AR, Lee TR. The Design of Maternity Monitoring System Using USN in Maternity Hospital. The Journal of Digital Policy \& Management. 2013.

[3] Cho GY, Lee SJ, Lee TR. Research on Enhancing Reliability of IT Convergence Technology Applied Emergency Management Information System. The Journal of Digital Policy \& Management. 2013.

[4] Galiano V, Lopez-Granado O, Malumbres MP, Migallon H. Fast 3D wavelet transform on multicore and many-core computing platforms. Journal of Supercomputing. 2013.

[5] Lee SJ, Roh YH, Kim YK, Lee TR. Geometric detection algorithm design for ECG data analysis using wavelet. International Journal of Bio-Science and Bio-Technology. 2013.

[6] Puthooran E, Anand RS, Mukherjee S. Lossless Compression of Medical Images Using a Dual Level DPCM with Context Adaptive Switching Neural Network Predictor. International Journal of Computational Intelligence Systems. 2013.

[7] Ji TY, Wu QH. Broadband noise suppression and feature identification of ECG waveforms using mathematical morphology and embedding theorem. Computer Methods and Programs in Biomedicine. 2013
[8] Rahman MZU, Karthik GVS, Fathima SY, Lay-Ekuakille A. An efficient cardiacsignal enhancement using time-frequency realization of leaky adaptive noise cancelers for remote health monitoring systems. Measurement. 2013.

[9] Salomon D. A Consice Introduction to Data Compression: Springer; 2008.

[10] Blelloch G. Introduction to Data Compression, 2001.

[11] Ziv J, Lempel A. UNIVERSAL ALGORITHM FOR SEQUENTIAL DATA COMPRESSION. Ieee Transactions on Information Theory. 1977.

[12] Ziv J, Lempel A. COMPRESSION OF INDIVIDUAL SEQUENCES VIA VARIABLE-RATE CODING. Ieee Transactions on Information Theory. 1978.

[13] Goldberger AL, Amaral LAN, Glass L, Hausdorff JM, Ivanov PC, Mark RG, et al. PhysioBank, PhysioToolkit, and PhysioNet: Components of a New Research Resource for Complex Physiologic Signals. Circulation. 2000.

[14] Chen M, Gonzalez S, Leung V, Zhang Q, Li M. A 2G-RFID-BASED E-HEALTHCARE SYSTEM. Ieee Wireless Communications. 2010.

[15] Touati F, Tabish R. U-Healthcare System: State-of-the-Art Review and Challenges. Journal of medical systems. 2013.

[16] Park S, KimW, Ihm I. Mobile collaborative medical display system. Computer Methods and Programs in Biomedicine. 2008.

[17] Sneha S, Varshney U. A framework for enabling patient monitoring via mobile ad hoc network. Decis Support Syst. 2013. 


\section{Cho Gyoun Yon}

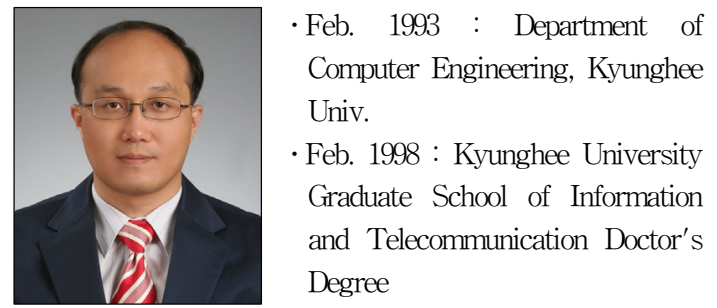

- Jan. $2000 \sim$ Jan. $2013:$ R\&D Center, Pantech Co. Ltd.

- Jul. $2013 \sim$ Present : Research Professor of Korea

University, Dept. of Healthcare Management Health

Science Institute

- Areas of Interest : Healthcare Information System, u-Healthcare, Healthcare Management, Mobile Communication, Mobile Network, Internet of Things

·E-Mail : gycho68@gmail.com

\section{Lee, Seo Joon}

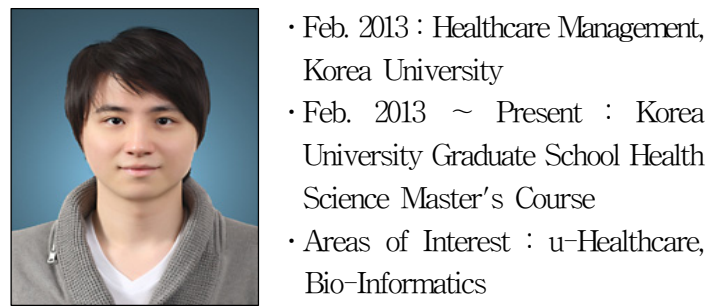

•E-Mail : richardlsj@korea.ac.kr

\section{Lee, Tae Ro}

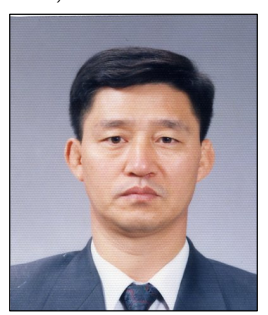

- Feb. 1984 : Department of Computer Engineering, Kwangwoon Univ.

- Aug. 1989 : Kyunghee University Graduate School of Education, Information and Telecommunication Master's Degree

Graduate School of Data Processing Doctor's Degree

- Sep. $2005 \sim$ Aug. 2006 : Visiting Professor of Griffith University

- Sep. 1996 Present : Professor of Korea University, Healthcare Management

- Areas of Interest : Healthcare Information System, $\mathrm{u}$-Healthcare, Management Information System, Signal Processing

·E-Mail : trlee@korea.ac.kr 